\title{
Hacia un plan local de prevención y atención del absentismo escolar en América Latina
}

\author{
Towards a Local Plan of Prevention and Attention of School Absenteeism in Latin America \\ Rumo a um plano local de prevenção e atenção à evasão escolar na América Latina
}

Alicia Cristina Razeto-Pavez

Pontificia Universidad Católica de Chile Santiago, Chile arazeto@uc.cl

https://orcid.org/0000-0003-2887-8424

Maribel García-Gracia Universidad Autónoma de Barcelona

Barcelona, España

maribel.garcia@uab.cat

https://orcid.org/0000-0001-6521-0437

Recibido • Received • Recebido: 05 / 12 / 2018

Corregido • Revised • Revisado: 01 / 05 / 2020

Aceptado • Accepted $\cdot$ Aprovado: 30 / 07 / 2020

\begin{abstract}
Resumen: El absentismo escolar es uno de los problemas socioeducativos que más crece en las escuelas de la región latino y centroamericana. Varios estudios demuestran sus efectos perjudiciales en el desempeño educativo del alumnado y en sus oportunidades futuras de desarrollo. Sin embargo, no se ha avanzado lo suficiente en la formulación de planes orientados a la intervención en la temática, que posicionen a las escuelas como responsables y al nivel local o territorial como escenario de las actuaciones, y de la movilización de recursos y agentes socioeducativos. El ensayo tiene por objetivo presentar recomendaciones para la formulación de planes locales de atención y prevención del absentismo escolar. Se centra en proponer condiciones básicas para la formulación de un plan local, como son el diseño de objetivos en sintonía con un propósito moral, un enfoque preventivo y un trabajo colectivo.
\end{abstract}

Palabras claves: Absentismo escolar; planificador de programas; escuela; derecho a la educación.

Abstract: School absenteeism is one of the most growing socio-educational problems in schools in the Latin and Central American region. Several studies show the harmful effects it has on the students' educational performance and their future development opportunities. However, insufficient progress has been made in the formulation of intervention-oriented plans on the subject, and schools are alleged to be responsible for this situation. They are also claimed as scenarios, at the local or territorial level, for actions and the mobilization of resources and socio-educational agents. The purpose of this essay is to present recommendations for the formulation of local plans address and prevent school absenteeism. It focuses on proposing basic conditions for the formulation of a local plan, such as the design of objectives in tune with a moral purpose, a preventive approach, and a collective work.

Keywords: School absenteeism; program planner; school; education rights. 
http://doi.org/10.15359/ree.24-3.28

http://www.una.ac.cr/educare

educare@una.ac.cr

\begin{abstract}
Resumo: A evasão escolar é um dos problemas socioeducativos que mais crescem nas escolas da América Latina e da América Central. Vários estudos mostram seus efeitos nocivos sobre o desempenho educacional de estudantes e suas oportunidades futuras de desenvolvimento. Entretanto, até agora os avanços na formulação de planos voltados à intervenção sobre o tema foram insuficientes, vendo as escolas como responsáveis e a nível local ou territorial como cenário de ações e mobilização de recursos e agentes socioeducativos. $O$ objetivo do estudo é apresentar recomendações para a formulação de planos locais de atendimento e prevenção da evasão escolar. Centra-se em propor condições básicas para a formulação de um plano local, como a elaboração de objetivos em sintonia com um propósito moral, uma abordagem preventiva e um trabalho coletivo
\end{abstract}

Palavras-chave: evasão escolar; planejador de programa; escola; direito à educação.

\title{
Introducción
}

El absentismo escolar crece en América Latina y el Caribe. Un estudio de la Organisation for Economic Cooperativon and Development (OECD) (2016) revela que, en México, un $26 \%$ del estudiantado faltó 1 día de clases al menos una vez en las dos semanas anteriores a la aplicación de la Prueba Pisa en 2015. En Costa Rica, la cifra asciende a un $39 \%$, en Perú a un 40 \%, en Colombia un $44 \%$ y en Uruguay un $52 \%$ de estudiantes. Estas cifras de absentismo aumentan en las escuelas más pobres y disminuyen en las más ricas (OECD, 2016).

El absentismo escolar hace referencia a la ausencia no justificada del alumnado matriculado a la escuela y se comprende como un proceso de ruptura temporal (absentismo puntual y regular) o permanente (absentismo crónico) con la escuela (García Gracia, 2013), Las situaciones de absentismo tienen múltiples causas y pueden expresar un proceso de desafección escolar, es decir, de falta de compromiso con la escuela (Appleton et al., 2008). Tal como señala Fernández Enguita (2011), este proceso de desenganche del estudiantado con la escuela es lento y progresivo, se inicia en primaria y se intensifica en secundaria. Una de las manifestaciones de este desenganche es el absentismo, pero también pueden serlo la indisciplina o los malos resultados académicos. Dado que es un proceso acumulativo, si es que no se revierte, en algunos casos más extremos el desenganche puede culminar en situaciones de absentismo crónico que se traducen en un abandono definitivo de la escuela (González González, 2005; Miñaca y Hervas, 2013).

Son varios los agentes que influencian los problemas escolares de desafección y fracaso escolar. Según Marchesi Ullastres (2003), no solamente están la disposición del alumnado y su contexto familiar, sino que también están el centro educativo -la forma en la que se organiza el aprendizaje y el currículo, la formación y estilo de enseñanza del profesorado-y el contexto sociocultural. Esta variedad de influencias le reviste complejidad a la intervención en las situaciones de absentismo escolar, y hace imprescindible contar con enfoques y dispositivos de intervención participativos, especializados, multinivel e interdisciplinarios. 
http://doi.org/10.15359/ree.24-3.28

De acuerdo con una lógica de derechos sociales, el absentismo escolar es un problema que debe ser abordado, ya que atenta contra uno de los derechos básicos de la infancia y juventud, como es el de tener la oportunidad de acceder y completar el ciclo educativo obligatorio provisto por cada legislación nacional. Tal como señala García Gracia (2009):

La erradicación del absentismo y del abandono escolar es uno de los retos fundamentales de nuestro sistema educativo si se pretende avanzar hacia una educación inclusiva que garantice la permanencia y continuidad con éxito de todo el alumnado. Se trata pues de un reto fundamental para mantener la cohesión social y reducir el riesgo de exclusión social que requiere de intervenciones holísticas e integrales. (p. 4)

En esta postura, el presente ensayo tiene por objetivos: i) comunicar la importancia que tiene la intervención en absentismo escolar, ii) destacar las posibilidades que ofrece la formulación de un plan local o municipal de prevención y atención del absentismo escolar, y ii) formular propuestas para el desarrollo de un plan.

La finalidad de este trabajo es influir en el contexto latinoamericano para la visibilización de la problemática del absentismo escolar y la puesta en marcha de planes locales, en vista de la creciente presencia de este fenómeno en los centros educativos de la región.

\section{¿Por qué intervenir en el absentismo escolar?}

Se presentan tres explicaciones que destacan la relevancia de desarrollar actuaciones para prevenir y atender este fenómeno socioeducativo en los centros educativos. La primera explicación enfatiza en los posibles efectos perjudiciales que tiene el absentismo en el presente y futuro desarrollo de los niños y las niñas. La segunda explicación describe las consecuencias que trae el absentismo al funcionamiento del sistema escolar y las políticas educativas. La tercera explicación ubica en la escuela la responsabilidad de aplicar medidas que le pongan atajo al absentismo, en tanto es el lugar en el que se manifiesta y es la misma escuela la que potencia dinámicas de absentismo.

\section{Efectos negativos en el aprendizaje y desarrollo de los niños, niñas y adolescentes}

La relevancia de abordar el absentismo escolar a través de programas de intervención, se basa en los datos disponibles que existen acerca de las consecuencias que tiene en el estudiantado. Una publicación de Balfanz y Byrnes (2012) reúne los resultados de un conjunto de estudios realizados en seis Estados de Estados Unidos sobre el impacto del absentismo crónico; concluyen que tiene efectos negativos en todos los niveles de enseñanza (preprimaria, primaria y secundaria) y que se resumen en que el absentismo crónico: 
http://doi.org/10.15359/ree.24-3.28

http://www.una.ac.cr/educare

educare@una.ac.cr

- Disminuye el desempeño del alumnado; se acentúa el hecho de provenir de familias de bajos ingresos; disminuyen las habilidades en matemáticas, lectura y escritura.

- Cuando se da en prekinder o kínder, aumenta la posibilidad de que se continúe durante la enseñanza primaria.

- Cuando se da en secundaria, además de afectar negativamente los resultados en pruebas en matemáticas y lectura, interrumpe y afecta las expectativas de los tiempos de graduación y aumenta las probabilidades del abandono educativo.

Un estudio realizado en California, Estados Unidos, por London et al. (2016) encontró que había una brecha continua a través de los años en los resultados de una prueba estandarizada de aprendizaje, en matemática y lenguaje, atribuible al absentismo crónico. No obstante, los efectos también trastocan al centro educativo, al profesorado, compañeros y compañeras de clase y a los equipos directivos. Estudios realizados por Gottfried (2011, 2015) muestran que el menor grado de desempeño en las pruebas estandarizadas también incluye al resto del alumnado de la clase. Esto también lo confirma un estudio la OECD (2016) a partir de los resultados Pisa 2015, el que señala que el desempeño en ciencia es peor no solo por parte del alumnado absentista, sino que de toda la clase. Durante el tiempo de ausencia, el grupo absentista ha perdido la oportunidad de interactuar con el resto de la clase. Además, se ve afectado por la desviación de tiempo y disrupción de la clase cuando retorna (Malcolm et al., 2003).

El absentismo quiebra la continuidad del aprendizaje, merma la autoconfianza del estudiantado (Malcolm et al., 2003). Produce menos resultados en aprendizaje de matemáticas y lectura; menos motivación con los estudios; menor adquisición de habilidades sociales, propias del desarrollo de un grupo social, e impacta en mayor dificultad para hacer y mantener amistades (Gottfried, 2014; Malcolm et al., 2003). Asimismo, el absentismo no solo afecta al alumnado, sino que también al profesorado. Este ve desviada su atención del resto de la clase y debe brindar de su tiempo libre para ayudar a los estudiantes y a las estudiantes absentistas para integrarles al aprendizaje (Malcolm et al., 2003). La situación de absentismo reporta incertidumbre, el profesorado, habitualmente, no conoce la razón de la ausencia ni su momento de reincorporación. Una vez que retorna, el profesorado puede sentirse frustrado o desmoralizado (Malcolm et al., 2003) por no saber cómo apoyar o no poder hacerlo como quisiera de modo tal de no perjudicar el avance personal ni el del grupo.

Estudios de absentismo escolar visibilizan la relación y el impacto que tiene en la salud de la infancia y de adolescentes, puesto que puede exponerles a riesgos para su desarrollo (Stempel et al., 2017). Estudios demuestran que el absentismo está asociado a la experiencia de vivir acontecimientos traumáticos, principalmente, la violencia en el barrio, el abuso de sustancias por parte de algún familiar y el maltrato (Melander et al., 2018; Stempel et al., 2017). El estudio de Melander et al., (2018) demostró que el 25 \% de estudiantes ausentes reportaron un tipo de maltrato, comparado al $16 \%$ del total de la población. 
http://doi.org/10.15359/ree.24-3.28

Finalmente, el absentismo escolar extiende su impacto a nivel social, porque no solo afecta las posibilidades de progresión escolar y culminación exitosa del ciclo educativo, sino que también las posibilidades futuras de empleo, promoción personal y profesional; puede acrecentar las probabilidades de marginación, paro, delincuencia, etc. (González González, 2005). Asimismo, este impacto no solo se reducirá al estudiantado absentista, sino que también a su núcleo familiar, al llegar a mermar el desarrollo futuro de sus miembros.

\section{Una amenaza al sistema educativo y una oportunidad para las políticas educativas}

El absentismo escolar pone en jaque a todo el sistema de educación público, puesto que este se basa en el principio de que el estudiantado asiste regularmente, día tras día, a la escuela, con excepción de la existencia de alguna enfermedad, visita al doctor, eventos especiales, crisis o vacaciones familiares (Balfanz y Byrnes, 2012). Sin la presencia del alumnado en el aula, no es posible el aprendizaje tal y como ha sido concebida por la escuela tradicional, en la cual existe un personal docente que enseña, a lo largo del año escolar y a través de diversas actividades guiadas por un currículo, a un grupo de estudiantes. Un absentismo exacerbado significaría el sinsentido y fracaso de la escuela y del proceso educativo.

Desde la perspectiva de la ciudadanía y de derechos sociales, propios de la política educativa, el absentismo imposibilita el cumplimiento del derecho a la educación. Tal como señala la Unesco (2016), la educación es un derecho humano fundamental y un derecho habilitador, y para hacerlo realidad "Ios países deben garantizar un acceso universal e igualitario a una educación y un aprendizaje inclusivos y equitativos de calidad, que deberán ser gratuitos y obligatorios, sin dejar a nadie rezagado" (p. 28). Es ineludible el rol garante del estado, puesto que la educación es un bien público. Pero también "la sociedad civil, los docentes y educadores, el sector privado, las comunidades, las familias, los jóvenes y los niños cumplen todos funciones clave para hacer efectivo el derecho a una educación de calidad" (Unesco, 2016, p. 28).

Contrariamente a estos ideales, el absentismo margina a la infancia y a la adolescencia de los procesos educativos y de sus positivos impactos futuros. Al mismo tiempo, imposibilita que estos grupos poblacionales cumplan sus derechos y sus funciones claves para hacer efectivo el derecho a la educación de calidad. Visto desde otra perspectiva, el absentismo desplaza el viejo problema del acceso a la educación hacia la equidad en la educación, pues a pesar de tener matrícula, estudiantes ausentes no ejercen el nivel de participación más básico y mínimo en el sistema educativo: estar presentes dentro del proceso de enseñanza y aprendizaje, y manifestar, así, su desapego frente a la institución educativa (Fernández Enguita, 2011) y un menor rendimiento escolar. 
http://doi.org/10.15359/ree.24-3.28

http://www.una.ac.cr/educare

educare@una.ac.cr

\section{Escuela como responsable principal}

Planificar e implementar actuaciones en torno al absentismo escolar es prioritario, si se considera que uno de los principales factores que lo produce se encuentra en el propio centro educativo, por sus prácticas pedagógicas y políticas socioeducativas (García Gracia, 2005). Hay varios estudios que demuestran que el proceso de desapego del estudiantado con la escuela se origina por relaciones conflictivas con el profesorado, compañeros o compañeras de clase o el ambiente en general del centro educativo. Así, por ejemplo, un estudio de Strand (2014) concluye que las principales razones que da el grupo de jóvenes a su ausencia se relacionan con factores del ambiente escolar, como la falta de relaciones sociales con sus pares y profesorado, no entender las instrucciones del profesorado, no tener amistades, tener conflictos en el colegio o aburrirse en clases. Otro estudio establece que hay factores relacionados con la escuela que influyen fuertemente en el absentismo, como lo es el bullying y el acoso escolar (Prakash et al., 2017). Este mismo aspecto, el ambiente escolar inseguro, también es indicado como un factor de riesgo de absentismo escolar por un estudio de Mallett (2016), quien agrega que un factor protector de la asistencia a clases es que la escuela cuente con un servicio de prevención de la inasistencia escolar, con estrategias de motivación para el alumnado de riesgo, y con un plan de alianza con agentes de la comunidad local (Mallett, 2016).

No resulta habitual que los centros educativos acepten ni comprendan que uno de los principales factores que incide en la aparición del absentismo provenga de sus propias prácticas. En realidad, prevalece la visión que centra la responsabilidad en las familias y el estudiantado, propio de la teoría del déficit o desventaja cultural y social (García Gracia, 2013), que centra su mirada en la falta de habilidades parentales y personales, creando una relación distanciada entre las familias y la escuela. A pesar de que los factores familiares y personales inciden de manera importante en las trayectorias educativas del alumnado absentista, el papel del centro educativo es central para evitar y revertir las relaciones de desapego con la escuela.

\section{Planes locales de intervención en absentismo escolar}

Han sido analizados los argumentos para comprender y apoyar las iniciativas de prevención y atención del absentismo escolar. Este ensayo propone una aproximación de intervención de nivel local o territorial. Lo local de un plan de intervención alude a la noción de territorio en el cual este es implementado. Habitualmente, se trata de un territorio de pequeña escala, delimitado en un barrio, comuna o municipalidad. Según García Gracia (2013), la noción de territorio supone superar la visión de unidad administrativa, para considerarlo como una unidad funcional de actuaciones basado en su identidad. Esta identidad consiste en un sentido de pertenencia de sus habitantes con ese territorio, en una manera de ser que les distingue de otros pueblos, que les hace potenciar sus capacidades y movilizarse para un proyecto común a favor del desarrollo de la sociedad local (Arocena, 2002). 
http://doi.org/10.15359/ree.24-3.28

De este modo, un plan local se origina desde un territorio que comparte cierta cultura, en el que sus habitantes son capaces de generar iniciativas locales en favor de un objetivo común. En el caso de un plan local en el ámbito educativo, la noción de territorio supone una dinámica de funcionamiento como zona escolar, es decir, una red de centros escolares, de servicios, y de recursos educativos y socioculturales (García Gracia, 2013).

Un plan local en el ámbito educacional requiere de un proceso de participación social en su diseño, implementación y evaluación, así como también de un agente que ejerza liderazgo para orientar el proceso de desarrollo. Los gobiernos locales o las municipalidades ${ }^{1}$ son la administración más próxima a las personas, por lo que pueden hacer un mejor y más preciso diagnóstico de las necesidades educativas de las personas (Federación Española de Municipio y Provincias [FEMP], 2018). Es fundamental, entonces, que el gobierno local lidere o potencie liderazgos para el proceso de formulación del plan, y que la red de centros escolares y de servicios y recursos educativos participe en el proceso de origen y desarrollo del plan local.

Formular un plan local no significa desatender el nivel central o gubernamental y el nivel del centro escolar. Esto sería paradójico, porque las reformas educativas se producen en el nivel central y se implementan en el escolar. Como señalan Barber y Fullan (2005), las reformas educativas sustentables se caracterizan por generar mejoras en tres niveles y en sus interrelaciones: el de la escuela, el del distrito (gobierno local o municipalidad) y el del Estado central. Se trata de una visión sistémica, un sistema de pensamiento que debe pasar a un sistema de acción.

Las actuaciones de las municipalidades en el tema del absentismo suelen provocarse por el mandato de la legislación nacional, en la que el Estado le otorga competencias al nivel local relativas al logro de los objetivos de las políticas educativas. En términos de política educativa, suelen estar enmarcados en las actuaciones en torno a la escolarización obligatoria, equidad educativa y el derecho a la educación.

En vista de su jerarquía intermedia entre el nivel central y el de la escuela, el mayor potencial del nivel municipal es ejercer liderazgo frente a las escuelas para orientarlas hacia el cometido del nivel central, apoyándolas en este proceso. En su tarea cotidiana, los gobiernos locales reconocen la importancia que tiene la educación para la prosperidad de los territorios y para lograr la cohesión social (FEMP, 2018).

Una de las principales herramientas de trabajo en el absentismo son los planes municipales Los planes municipales contra el absentismo escolar, son concebidos como un dispositivo local de intervención, es decir, como un mecanismo articulado en el territorio diseñado para

\footnotetext{
${ }^{1}$ En Chile, las municipalidades o municipios son las instituciones que administran localmente las comunas. Su principal finalidad es satisfacer las necesidades de la comunidad local y asegurar su participación en el progreso económico, social y cultural.
} 
http://doi.org/10.15359/ree.24-3.28

http://www.una.ac.cr/educare

educare@una.ac.cr

combatir el absentismo escolar mediante actuaciones de prevención, detección, reparación y seguimiento de situaciones de absentismo, así como la evaluación de la eficacia de las intervenciones desarrolladas por la mejora del dispositivo. (p. 101)

En este ensayo se ha determinado que es la escuela la principal responsable para desarrollar las iniciativas de atención y prevención del absentismo escolar. No obstante, también se ha propuesto que, debido a la complejidad y diversidad del del fenómeno, no es posible pensar en estrategias de abordaje aisladas, sino que se requiere de un trabajo colectivo y articulado con otros agentes, liderados por un agente que puede ser el municipio. Se trata de poner en marcha el principio de corresponsabilidad.

García Gracia (2013) propone a la corresponsabilidad como uno de los pilares rectores para la implementación de un programa de reducción del absentismo escolar. Según García Gracia (2013):

La corresponsabilidad es un principio y criterio de actuación que presupone un compromiso compartido entre diferentes administraciones para el cumplimiento de los deberes y obligaciones relativos a la garantía del derecho a la educación. Se fundamenta en el compromiso, cooperación y participación desde la proximidad, de los diferentes agentes educativos. (p. 128)

Por corresponsabilidad de la comunidad educativa se refiere a aquella que se puede producir (García Gracia, 2013):

- Entre instituciones tales como la escuela, familia y organizaciones comunitarias

- Entre administraciones educativas a nivel nacional, regional y local con la finalidad de integrar políticas y buscar sinergias entre programas, recursos y servicios en el territorio.

- Entre departamentos o áreas de las mismas administraciones, a fin de garantizar la eficacia y eficiencia de los programas y recursos, y la coordinación de las actuaciones.

\section{Condiciones para la formulación de un plan local de prevención y atención del absentismo escolar}

El diseño e implementación de un plan local de prevención del absentismo requiere algunas condiciones previas para garantizar su eficacia. Este ensayo identifica tres condiciones necesarias detectadas a partir de la revisión bibliográfica y considera que el éxito de un plan pasa por la integralidad de las actuaciones. Ello requiere de un trabajo articulado 
entre los diversos agentes que intervienen en su implementación, particularmente entre profesionales y personal técnico de educación, y de la intervención social en los centros educativos. (García Gracia, 2013)

\section{Objetivos en sintonía con un propósito moral}

Los objetivos de un plan local consideran que los centros educativos pueden ser muy distintos entre sí, aunque estén emplazados en un mismo territorio, y compartan cierta historia, identidad y propósito común. Esta diferencia proviene tanto de las características socioculturales del alumnado y de sus familias, como también de las características organizacionales del centro educativo, su historia, su estructura, dinámicas y culturas organizacionales. En vista de esta diversidad, los objetivos de un plan local de absentismo se orientan hacia la mejora del éxito en las escuelas, a fin de prevenir y reconducir los procesos de desenganchey absentismo. Estos objetivos se concretan, de manera específica, en cada escuela, en el marco de la autonomía de los centros.

En los procesos de reformas educativas, es fundamental que los diversos actores compartan un propósito moral (Barber y Fullan, 2005) que les convoque y motive a procesar los cambios y desempeñarse de la mejor manera, a pesar de las frustraciones. Según Barber y Fullan (2005), este propósito moral suele ser la mejora del logro del estudiantado y la disminución de las brechas de desigualdad educativa. Los objetivos de un plan de absentismo tienen que estar fundados en un propósito común que puede ser el logro de la escolaridad obligatoria, el éxito escolar, la calidad de la educación o el derecho a la educación, dependiendo de lo que consensuen los actores en el proceso participativo, lo que establezca la legislación o las prioridades de las agendas gubernamentales. Es fundamental que la formulación de los objetivos del plan sea de manera participativa, de modo tal que los diferentes agentes expresen sus motivaciones y sus valores en relación con el absentismo. La determinación del propósito moral se originará producto de este proceso de deliberación colectiva y de los objetivos que la política educativa y la legislación nacional establezcan.

\section{Un enfoque preventivo}

La prevención del absentismo escolar es un eje central y estratégico del plan. Tiene por objetivo que las escuelas y recursos territoriales se anticipen al surgimiento de nuevos casos de absentismo, evitándolos a través de intervenciones oportunas y permanentes durante el curso escolar. Tal como lo señala el estudio de Kearney y Graczyk (2014), el diseño de una intervención efectiva para promover la asistencia a clases pasa por una identificación temprana y una pronta intervención, evita que los casos acumulen un largo número de ausencias, pues se cronifica el absentismo, lo que hace más difícil reconducir estas situaciones.

Uno de los principales focos de la prevención del absentismo tiene que ver con promover el sentido de pertenencia y el enganche del alumnado con el centro educativo (Cook et al, 2017), lo que supone intervenciones sistemáticas de la escuela durante el año escolar y que 
http://doi.org/10.15359/ree.24-3.28

http://www.una.ac.cr/educare

educare@una.ac.cr

involucren a toda la comunidad educativa, para desarrollar un buen clima escolar y un buen sentido de acogida para el alumnado por parte de la escuela. En definitiva, la prevención no es un desafío sencillo y debe ser realizado por la escuela en alianza con otros centros educativos y recursos socioeducativos del territorio, y con todos los agentes implicados (familia, comunidad, profesorado), tal como lo sugiere una investigación de Miñaca y Hervas (2013). Un plan local debe instar a las escuelas a desarrollar actividades en alianza con estos actores, tendiendo a promover su compromiso con el proceso educativo de los niños, niñas y jóvenes. Por eso mismo, el plan local de absentismo también debe articularse y complementarse con otros planes y programas existentes en el territorio y en los centros educativos, orientados a favorecer el enganche de sus estudiantes con la escuela, tales como los vinculados a la convivencia escolar, la diversidad, entre otros.

Un proceso de intervención central en la estrategia preventiva es la detección precoz y oportuna de los posibles casos de absentismo. La detección eficaz es una acción del centro educativo e implica el diseño y utilización de algún instrumento que mida variables de riesgo de absentismo y abandono del estudiantado y su familia, que puede incluir variables de engagement escolar y también las relativas a las condiciones de vida y de escolaridad. El manejo responsable y oportuno de estos datos puede permitir que la escuela formule acciones de mejora y de enganche, revierta el absentismo y logre una prevención eficaz.

Por otra parte, está la tarea de la detección y registro una vez que el absentismo ya se ha presentado. En un plan local se debiera instar a que las escuelas cuenten con proceso e instrumentos de detección y registro comunes, para visibilizar la frecuencia de la inasistencia y producir información fiable que posibilite la intervención precoz. El control sistemático de la asistencia es una estrategia importante, pues permite observar irregularidades y conseguir una detección rápida, que permita actuar inmediatamente en las primeras fases del absentismo.

El proceso de detección y registro genera datos, los que permiten visibilizar el absentismo escolar como una problemática real, ubicándola como una prioridad para las políticas educativas. En definitiva, es importante transmitir a los centros educativos que la detección y registro no es una tarea o exigencia adicional a las ya asumidas, sino que supone una actuación en favor del bienestar presente y oportunidades de desarrollo futuro de la población infantil y adolescente.

No solo es importante el compromiso y la formación de cada miembro de la escuela, sino que también es necesaria la definición de responsabilidades para esta labor, para resolver la pregunta de quién o quiénes detectan y registran; además, de cómo lo realizarán.

Los diversos mecanismos contemplados en la estrategia preventiva consideran las transiciones educativas como uno de los momentos vulnerables al absentismo escolar. Las transiciones entre los niveles de enseñanza pueden generar desapego con la escuela; por los cambios e incertidumbres que conlleva, aumentan los riesgos de absentismo escolar e incluso del abandono (García Gracia, 2013; Gimeno Sacristán, 1997; Monarca y Rincón Gallego, 2010; 
Monarca et al. 2012). De este modo, a la detección del absentismo se le suma la necesidad de incorporar mecanismos de orientación del acompañamiento al alumnado y familias, tanto de orden psicosocial como educativo, con miras a favorecer la adaptación con la escuela y sus procesos de aprendizaje (Ross, 2016).

Otro mecanismo relevante dentro de la estrategia preventiva es favorecer el retorno y la adaptación del alumnado absentista que se reincorpora a clases, a través de la implementación de un plan de retorno y acogida (García Gracia, 2013). Este plan contribuye a prevenir el absentismo crónico o a evitar que su desenlace sea el abandono. La herramienta organiza acciones para facilitar la adaptación del niño o niña a la escuela, flexibilizando mecanismos y plazos para su adecuada reintegración y mayor enganche con el centro educativo. Ello enfatiza la importancia de la comunicación interna del regreso a clase entre el personal docente de la escuela, de modo tal que le reciban con afecto y eviten formular comentarios o preguntas incomodas. Por otra parte, organizar un plan de retorno que, en su inicio, será flexible en horarios de ingreso o cumplimiento de obligaciones académicas, si así fuese necesario, incentiva un acuerdo entre la escuela, el estudiantado y su familia.

\section{Un trabajo colectivo}

Una de las claves de éxito de los programas contra el absentismo es "la prevención, la información, la formación y la sensibilización de todas las personas que conforman la comunidad educativa desde la enseñanza primaria" (FEMP, 2018, p. 10). De esta forma, un trabajo en equipo, dialogante, colaborativo e interdisciplinario es central para lograr formular e implementar un plan local de prevención y atención del absentismo (García Gracia, 2013). Las múltiples dimensiones y agentes implicados en el fenómeno del absentismo escolar subrayan la necesidad de contar con equipos de trabajo colectivos, diversos y comprometidos, orientados a alcanzar los objetivos del plan y motivados por su propósito moral.

El trabajo colegiado materializa el principio de corresponsabilidad planteado en la sección anterior, a través del cual diferentes agentes cooperan y participan para el logro del derecho a la educación. Esta forma de trabajo implica comprender que, si bien la escuela tiene una responsabilidad en el origen y la solución del absentismo, no puede desarrollar estrategias de abordaje aisladas: se requiere de la participación y compromiso de otros agentes (Gase et al., 2015). Requiere de un trabajo en alianza con otros centros educativos del territorio, de otros agentes y recursos socioeducativos, con los cuales poder complementar las estrategias de prevención y atención del absentismo. Estos agentes y recursos pueden corresponder a:

- Área de servicios educativos y de servicios sociales del municipio o ayuntamiento.

- Fundaciones u organizaciones no gubernamentales del área socioeducativa. 
http://doi.org/10.15359/ree.24-3.28

http://www.una.ac.cr/educare

educare@una.ac.cr

- Centros de salud.

- Servicios educativos descentralizados en el territorio del nivel central

- Policía local

La eficacia de un trabajo colectivo supone la definición de responsabilidades y de una estructura, que implica una manera de denominar y hacer visible ese trabajo colaborativo. Una alternativa interesante es el de la figura de las comisiones, existente en experiencias internacionales (García Gracia, 2013). La eficacia de un trabajo a través de comisiones es que estas sean predominantemente técnicas, ejecutivas, centradas en el abordaje de los casos de absentismo y en las estrategias preventivas, y no políticas ni puramente discursivas. Para ello, contarán con un plan y herramientas de trabajo y con procesos evaluativos.

Un plan local de absentismo, inspirado en el principio de la corresponsabilidad, puede promover la creación de dos comisiones en torno al absentismo, una que se conforma en el centro educativo, y otra que se conforma a nivel territorial o local. La primera agrupa al equipo del centro educativo, que puede asumir la responsabilidad de planificar las acciones preventivas, y el diagnóstico y evaluación de los indicadores de riesgo o de los casos de absentismo, y las primeras intervenciones con el alumnado y sus familias. El equipo del centro educativo tiene una gran centralidad, puesto que es el que más conoce al estudiantado y más tiempo tiene de relación con este. Esto podría predecir un mejor vínculo y mejores resultados de la intervención. Por otra parte, este equipo podrá emprender acciones para mejorar los factores escolares que desencadenan absentismo.

La segunda comisión, de nivel municipal o territorial, puede estar compuesta por una red de diversos organismos públicos y privados que tienen relación con la escolarización y el absentismo, incluyendo al centro escolar. Estas comisiones son una instancia local que agrupa a los centros educativos y a representantes de diferentes organismos sociales y educativos que tienen algún grado de responsabilidad relativa al absentismo escolar y el logro de la plena escolarización. Su principal finalidad es movilizar recursos territoriales para realizar prevención y atender casos complejos de absentismo, que no logran ser solucionados por el equipo de trabajo del centro escolar.

\section{Conclusiones}

El absentismo escolar puede perjudicar el desarrollo de la infancia y adolescencia, no solo en el ámbito educativo, sino también en el social. Considerando esta relación, el ensayo contribuye a transmitir la importancia que tiene formular planes de intervención orientados a prevenir y atender esta problemática; sitúa, en el ámbito territorial, el desafío de formular un plan local que oriente a los centros educativos y recursos territoriales a trabajar en conjunto. El 
http://doi.org/10.15359/ree.24-3.28

ámbito del territorio genera oportunidades y ventajas que son precisas de valorar y utilizar en la intervención en absentismo, aunque el núcleo del proceso de cambio se ubica al interior del centro educativo. La principal ventaja es que los centros educativos comparten cierta identidad sociocultural, la cual puede incentivar el diálogo al reconocerse en condiciones cotidianas de trabajo similares. Asimismo, esta identidad les permite construir colectivamente alternativas de acciones en torno a sus problemas, al tener recursos similares en el territorio.

En el nivel local, el municipio puede asumir liderazgos centrales en la formulación y acompañamiento de la implementación de un plan local de absentismo. Esta es una oportunidad, puesto que los centros educativos tienen un exigente trabajo pedagógico, permeado por la cotidianeidad, el cual les dificulta agregar otros focos de acción socioeducativas. En tanto, para el municipio, un plan local de absentismo representa una oportunidad para cumplir su mandato legal vinculado a la administración del ámbito educacional.

El proceso de formulación e implementación de un plan local tiene ciertos requisitos que facilitarán su aplicación en el centro escolar y su impacto en la reducción del absentismo. En primer lugar, está el requisito de la participación, que sea formulado a través de un proceso abierto y dialogante con sus principales agentes: los centros educativos y los recursos socioeducativos del territorio. En segundo lugar, está el requisito de la difusión, que sea permanentemente diseminado en estos agentes a lo largo del territorio y del tiempo, teniendo en cuenta que los centros educativos presentan múltiples exigencias y constantes cambios en su personal. En tercer lugar, está el requisito de la flexibilidad del plan, para que los centros educativos puedan adaptarlo a su propia realidad, a sus dinámicas y estructuras organizativas, a sus capacidades. Por último, el requisito de la evaluación, puesto que el proceso de adaptación del plan local podría dar origen a un plan de trabajo específico en cada centro, con metas precisas y evaluables.

Finalmente, si bien el nivel local presenta ventajas indiscutibles para avanzar en la prevención y abordaje del absentismo, en Estados unitarios y centralistas como los que predominan en América Latina, se necesita un nivel central que haya priorizado la incorporación del tema en la agenda pública y se haya comprometido con el desarrollo de una política pública social y educativa.

\section{Referencias}

Appleton, J. J., Christenson, S. L. y Furlong, M. J. (2008). Student engagement with school: Critical conceptual and methodological issues of the construct. Psychology in Schools, 45(5), 369386. http://dx.doi.org/10.1002/pits.20303

Arocena, J. (2002). El desarrollo local. Un desafío contemporáneo. Editorial Taurus; Universidad Católica. 
http://doi.org/10.15359/ree.24-3.28

http://www.una.ac.cr/educare

educare@una.ac.cr

Balfanz, R. y Byrnes, V. (2012). Chronic Absenteeism: Summarizing what we know from nationally available data. Johns Hopkins University Center for Social Organization of Schools.

Barber, M. y Fullan, M. (2005) 'Tri-Level development' Putting systems thinking into action. Education Week. https://www.edweek.org/ew/articles/2005/03/02/25fullan.h24.html

Cook, P. J., Dodge, K. A., Gifford, E. J. y Schulting, A. B. (2017). A new program to prevent primary school absenteeism: Results of a pilot study in five schools. Children and Youth Services Review, 82, 262-270. https://doi.org/10.1016/j.childyouth.2017.09.017

Federación Española de Municipio y Provincias. (2018). III Catálogo de buenas prácticas municipales en la prevención y atención del absentismo y del acoso escolar. http://recs.es/iii-catalogo-debuenas-practicas-municipales-en-la-prevencion-y-atencion-del-absentismo-y-del-acosoescolar/

Fernández Enguita, M. (2011). Del desapego al desenganche y de este al fracaso escolar. Cadernos de pesquisa, 41(144), 732-751. https://doi.org/10.1590/S0100-15742011000300005

García Gracia, M. (2005). Culturas de enseñanza y absentismo escolar en la enseñanza secundaria obligatoria: Estudio de casos en la ciudad de Barcelona. Revista de educación, 338, 347374. http://www.educacionyfp.gob.es/revista-de-educacion/numeros-revista-educacion/ numeros-anteriores/2005/re338.html

García Gracia, M. (2009). El absentismo escolar: Algunas claves para el desarrollo de intervenciones integradas en el marco de una escuela inclusiva y del territorio. Monográficos escuela, 4-6. https://www.researchgate.net/profile/Antonio Bolivar/ publication/286884137 Absentismo y abandono escolar Politicas y buenas practicas/links/566ee8c108ae4d4dc8f7d999.pdf

García Gracia, M. (2013). Absentismo y abandono escolar. Editorial Síntesis.

Gase, L. N., Butler, K. y Kuo, T. (2015). The current state of truancy reduction programs and opportunities for enhancement in Los Angeles County. Children and Youth Services Review, 52, 17-25. https://doi.org/10.1016/j.childyouth.2015.02.008

Gimeno Sacristán, J. (1997). La transición a la educación secundaria. Discontinuidades en las culturas escolares. Editorial Morata.

González González, M. T. (2005). El absentismo y el abandono: Una forma de exclusión escolar. Revista de curriculum y formación de profesorado, 9(1), 1-12. https://recyt.fecyt.es/index. php/profesorado/article/view/42396/24325

Gottfried, M. A. (2011). Absent peers in elementary years: The negative classroom effects of unexcused absences on standardized testing outcomes. Teachers College Record, 113(8), $1597-1632$. 
http://doi.org/10.15359/ree.24-3.28

Gottfried, M. A. (2014). Chronic absenteeism and its effects on students' academic and socioemotional outcomes. Journal of Education for Students Placed at Risk, 19(2), 53-75. http://dx.doi.org/10.1080/10824669.2014.962696

Gottfried, M. A. (2015). Chronic absenteeism in the classroom context: Effects on achievement. Urban Education, 54(1), 3-34. http://dx.doi.org/10.1177/0042085915618709

Kearney, C. A. y Graczyk, P. (2014). A response to intervention model to promote school attendance and decrease school absenteeism. Child \& Youth Care Forum, 43(1), 1-25. https://doi.org/10.1007/s10566-013-9222-1

London, R. A., Sanchez, M. y Castrechini, S. (2016). The dynamics of chronic absence and student achievement. Education Policy Analysis Archives, 24(112), 1-27. https://epaa.asu.edu/ojs/ article/view/2471/1843

Malcolm, H., Wilson, V., Davidson, J. y Kirk, D. (2003). Absence from school: A study of its causes and effects in seven LEAs (Research Report No 424). Department for Education and Skills. http:// dera.ioe.ac.uk/8655/1/RR424.pdf

Mallett, C. A. (2016), Truancy: It's not about skipping school. Child and Adolescent Social Work Journal, 33(4), 337-347. https://doi.org/10.1007/s10560-015-0433-1

Marchesi Ullastres, A. (2003). El fracaso escolar en España (Documento de trabajo 11). Fundacón Alternativas. $\quad$ http://www.fundacionalternativas.org/public/storage/laboratorio documentos archivos/xmlimport-ZPRIx1.pdf

Melander Hagborg, J., Berglund, K. y Fahlke, C. (2018). Evidence for a relationship between child maltreatment and absenteeism among high-school students in Sweden. Child abuse \& neglect, 75, 41-49. https://doi.org/10.1016/j.chiabu.2017.08.027

Miñaca, M. I. y Hervas, M. (2013). Intervenciones dirigidas a la prevención del fracaso y abandono escolar: Un estudio de revisión. Revista Española de Educación Comparada, 21, 203-220. https://doi.org/10.5944/reec.21.2013.7620

Monarca, H. y Rincón Gallego, J. (2010). Tránsito a la ESO, ¿continuidad o ruptura? Cuadernos de Pedagogía, 401, 28-30. https://iesrdguezmonino.educarex.es/recursos/orientacion/ TRANSITOP.pdf

Monarca, H. A., Rappoport, S. y Fernández González, A. (2012). Factores condicionantes de las trayectorias escolares en la transición entre enseñanza primaria y secundaria. REOP, 23(3), 49-62. https://doi.org/10.5944/reop.vol.23.num.3.2012.11461

Organisation for Economic Cooperativon and Development. (2016). PISA 2015. Results excellence and equity in education (Vol. 1). Autor. https://www.oecd-ilibrary.org/education/pisa-2015results-volume-i 9789264266490-en 
http://doi.org/10.15359/ree.24-3.28

http://www.una.ac.cr/educare

educare@una.ac.cr

Prakash, R., Beattie, T., Javalkar, P., Bhattacharjee, P., Ramanaik, S., Thalinja, R., Murthy, S., Davey, C., Blanchard, J., Watts, C., Collumbien, M., Moses, S., Heise, L. e Isac, S. (2017). Correlates of school dropout and absenteeism among adolescent girls from marginalized community in north Karnataka, south India. Journal of adolescence, 61, 64-76. https://doi.org/10.1016/j. adolescence.2017.09.007

Ross, T. (2016). The differential effects of parental involvement on high school completion and postsecondary attendance. Education Policy Analysis Archives, 24(30), 1-38. https://epaa. asu.edu/ojs/article/view/2030

Stempel, H., Cox-Martin, M., Bronsert, M., Dickinson, LK. M. y Mandy, M. A. (2017). Chronic School Absenteeism and the Role of Adverse Childhood Experiences, Academic pediatrics, 17(8), 837-843. https://doi.org/10.1016/j.acap.2017.09.013

Strand, A.-S. M. (2014). 'School - no thanks - it ain't my thing': Accounts for truancy. Students' perspectives on their truancy and school lives. International Journal of Adolescence and Youth, 19(2), 262-277. https://doi.org/10.1080/02673843.2012.743920

Unesco. (2016). Educación 2030. Declaración de Incheon y marco de acción para la realización del objetivo de desarrollo sostenible 4. http://unesdoc.unesco.org/ images/0024/002456/245656s.pdf 